\title{
UV radiation-induced skin aging in hairless mice is effectively prevented by oral intake of sea buckthorn (Hippophae rhamnoides L.) fruit blend for 6 weeks through MMP suppression and increase of SOD activity
}

\author{
IN SIK HWANG ${ }^{1}$, JI EUN KIM ${ }^{1}$, SUN IL CHOI ${ }^{1}$, HYE RYUN LEE ${ }^{1}$, YOUNG JU LEE ${ }^{1}$, MIN JU JANG ${ }^{2}$, \\ HONG JU SON ${ }^{1,2}$, HEE SEOB LEE ${ }^{2,3}$, CHUNG HUN OH ${ }^{4}$, BAE HWAN KIM ${ }^{5}$, \\ SANG HAK LEE ${ }^{1,2}$ and DAE YOUN HWANG ${ }^{1,2}$ \\ ${ }^{1}$ College of Natural Resources and Life Science, ${ }^{2}$ Wellbeing Products RIS Center, Pusan National University, \\ Miryang 627-706; ${ }^{3}$ College of Human Ecology, Pusan National University, Busan 627-706; ${ }^{4}$ School of Dentistry, \\ Dankook University, Cheonan 330-714; ${ }^{5}$ Keimyung University, Daegu 704-701, Republic of Korea
}

Received March 2, 2012; Accepted April 27, 2012

DOI: $10.3892 / \mathrm{ijmm} .2012 .1011$

\begin{abstract}
Oxidative stress and oxidative photodamage induced by UV radiation can cause serious skin damage that is characterized by wrinkling, roughness, laxity and pigmentation. The effects of a sea buckthorn (Hippophae rhamnoides L.) fruit blend (SFB) containing sea buckthorn fruit extract, blueberry extract and collagen on UV-induced skin aging were examined by treating hairless mice for 6 weeks with UV irradiation and SFB administered orally. The effects of SFB were measured in the skin of these mice by phenotypical and histological analysis and western blotting. According to wrinkle formation analysis, the oral intake of SFB induced a decrease in wrinkle formation in the damaged skin of UV-irradiated mice. The thickness of the epidermis and dermis in the vitamin extracts (Vit)- and SFB-treated group was lower than that in the vehicle-treated group, but the group treated with SFB50 was the most effective group. The mice treated with the Vit- or SFB solution maintained a normal moisture content through the inhibition of transdermal water loss (TEWL) and an increase in skin moisture content. Furthermore, the levels of matrix metalloproteinase (MMP) and collagen protein expression were assessed in five groups to examine the mechanisms underlying the effects of SFB oral intake. The application of SFB induced a decrease in MMP-1 and -9 expression to the levels observed in the vehicle-treated group, but MMP-9 expression showed a much larger decrease
\end{abstract}

Correspondence to: Dr Dae Youn Hwang, Department of Biomaterial Science, College of Natural Resources and Life Science, Pusan National University, 50 Cheonghak-ri, Samnangjin-eup Miryang-si, Gyeongsangnam-do 627-706, Republic of Korea

E-mail: dyhwang@pusan.ac.kr

Key words: sea buckthorn fruit, collagen, photoaging, matrix metalloproteinase, superoxide dismutase than MMP-1. Furthermore, the expression of collagen-1 in the skin corresponded to MMP expression except for the SFB30-treated group, whereas the superoxide dismutase (SOD) activity was increased dramatically in the SFB50treated group. These results suggest that SFB has potential as a protective and therapeutic drug candidate against skin aging that functions by regulating the moisture content, MMP expression levels and SOD activity.

\section{Introduction}

The major cause of skin aging is both the passage of time (intrinsic ageing) and cumulative exposure to external influences (extrinsic ageing), such as UV radiation and smoking $(1,2)$. Intrinsically aged skin is characterized by fine wrinkling and reduced elasticity, whereas extrinsically-aged skin exposed to UV light is associated with the induction of both deep wrinkles and a significant loss of elasticity $(3,4)$. Of the range of external factors inducing skin ageing, UV radiation is considered a key cause of skin damage, which is characterized by deep wrinkles, roughness, laxity and pigmentation (5). In addition, these phenotypical changes in the skin induced by direct exposure to UV light are tightly associated with a significant increase in the concentration of reactive oxygen species (ROS) (6-8). An increase in ROS generation can help destroy the intracellular antioxidant-defense mechanism, as well as cause oxidative photodamage and oxidative stress to the cellular constituents including DNA, lipids or proteins in skin tissue $(9,10)$. In particular, oxidative stress plays an important role in initiating and driving the cascade events that induce the cell responses following exposure to UV radiation $(11,12)$.

Many of the antioxidants and anti-photoaging compounds, which act effectively against photodamage of the skin, are available. The methanol extracts of Corallina pilulifera exhibit antioxidative activity and have a protective effect on UVA-induced oxidative stress in human dermal fibroblasts (13). In addition, esculetin isolated from Fraxinus chinensis was 
reported to exhibit the strongest scavenging activity against 1,1-diphenyl-2-picrylhydrazyl (DPPH) and free radical scavenging activity in UVB-irradiated human dermal fibroblasts (14). A series of 2,2'-dithiocinnamate derivatives (DTCD) and 2,2'-dithio (DTBD) or 2-thiobenzoate derivatives (TBD) were synthesized as new anti-photoaging agents, which exhibited radical scavenging activity and MMP-1 inhibitory activity (15). On the other hand, little has been reported regarding the in vivo effects of specific blends involving a sea buckthorn fruit extract on the homeostasis and photodamage of skin although sea buckthorn has shown a potential therapeutic effect on skin, lung and gastric disease.

Therefore, the present study examined the effects of the oral intake of SFB on the fundamental properties of hairless mice skin including wrinkle formation, skin water content, collagen content and antioxidant status. These results provide strong evidence of the potential of SFB in the prevention or alleviation of UV-induced skin aging.

\section{Materials and methods}

Preparation of SFB. SFB was prepared from food-grade aqueous extracts of five components, sea buckthorn fruit extract, blueberry extract, collagen, hyaluronic acid and pure natural honey. Table I lists the source and content (\%) of SFB. To prepare the SFB solution, the five components were well mixed at $90^{\circ} \mathrm{C}$ for $10 \mathrm{~min}$ and filtered through $3 \mathrm{M}$ paper. Before the treatment, SFB was diluted with $\mathrm{dH}_{2} \mathrm{O}$ to make two dilute solutions (SFB30 and SFB50) with different concentrations (30 and 50\%). A Vit which was used as the control, was prepared by adding only a $21.3 \%$ extract of sea buckthorn fruit.

Care and use of animals. The animal protocol used in this study was reviewed and approved based on ethical procedures and scientific care by the Pusan National University-Institutional Animal Care and Use Committee (PNU-IACUC; approval number PNU-2011-00198). Adult HR-1 hairless mice were purchased from Central Lab. Animal, Inc. (Seoul, Korea) and handled at the Pusan National University Laboratory Animal Resources Center accredited by Korea FDA (unit number000996). All mice were given a standard irradiated chow diet (Purina Mills, Seongnam, Korea) ad libitum, and were maintained in a specific pathogen-free (SPF) state under a strict light cycle (light on at $06: 00 \mathrm{~h}$ and off at $18: 00 \mathrm{~h}$ ) at $22 \pm 2^{\circ} \mathrm{C}$ and $50 \%$ relative humidity.

Experimental design and UV radiation. Eight-week-old hairless mice $(n=35)$ were assigned to one of five groups $(n=7$ per group): a no-radiation group (no group), vehicle-treated group (vehicle group), vitamin-treated group (Vit group), SFB30-treated group (SFB30 group) and SFB50-treated group (SFB50 group). The four groups, either than the no-radiation group, were exposed to UV light using a UV radiation device for 6 weeks, and the mice were given access to the various solutions ad libitum. The vehicle-treated group was given a consistent volume of water daily, whereas the treatment groups were given Vit, SFB30 or SFB50 diluted with distilled water. The body weights of each mouse were measured using a chemical balance every week. At 6 weeks after commencing the vehicle, Vit and SFB treatments, the animals were sacri- ficed immediately using $\mathrm{CO}_{2}$ gas to acquire blood and skin tissue samples. The samples were stored in Eppendorf tubes at $-70^{\circ} \mathrm{C}$ until assayed.

$U V$ radiation. The minimal erythemal dose (MED) from the UV irradiation device was determined using the procedures suggested in previous studies $(16,17)$. A UV irradiation device was made from a TL20W/12RS UV lamp and Kodacel filter in a rectangular parallelepiped box. The UV lamp (Philips, The Netherlands) had an emission spectrum between 274 and $380 \mathrm{~nm}$, which was composed of 10.2\% UVC (275-290 nm), $53.5 \%$ UVB (290-320 nm), 25.3\% UVA1 (320-340 nm) and 11.2\% UVA2 (340-380 nm). Kodacel Sheeting 6805 Product (Kodak, USA) was used to remove UVC wavelengths $<290 \mathrm{~nm}$ in front of the UV lamp. The irradiation intensity was measured at $30 \mathrm{~cm}$ from the light source using a UVX Radiometer (UVP, USA). To determine the 1MED, the dorsal skin of mice was exposed to different doses of UV light and the formation of erythema was detected after $24 \mathrm{~h}$. Skin aging was induced by irradiation of 1MED three times per week (Monday, Wednesday and Friday) for 6 weeks.

Evaluation of wrinkle formation. Wrinkle formation was measured using the procedure established by our laboratory using a DETAX System II (MIXPAC) and Double-Stick Disc (3M Health Care, Germany) (17). After 6 weeks, skin surface impressions (replica) were prepared by applying silicon rubber in mixed liquid form secreted from a DETAX System II to the dorsal skin of the mice. The depth, number of wrinkles on each skin impression was analyzed and classified into one of the four degrees suggested by Bissett et al (18). In this analysis, grade 0 indicated no wrinkle formation, grade 1 indicated some shallow wrinkles, grade 2 indicated some wrinkles, and grade 3 indicated several deep wrinkles (19).

Measurement of the skin moisture content, transepidermal water loss (TEWL) and erythema dose. All three factors related to skin homeostasis were assessed on the dorsal skin of each mouse using the appropriate devices (20). TEWL was detected using a Corneometer CM825 (Courage and Khazaka Electronics, Cologne, Germany). In addition, the skin moisture content was measured using a Tewameter TM300, and the erythema dose was analyzed using a Mexameter MX18 (all were from Courage and Khazaka Electronics) according to the manufacturer's protocol. Each detection was performed 3 times on every site on the dorsal skin of hairless mice.

Western blotting. The proteins prepared from the skin tissues of the vehicle, Vit or SFB-treated mice were separated by 4-20\% sodium dodecyl sulfate-polyacrylamide gel electrophoresis (SDS-PAGE) for $3 \mathrm{~h}$, and the resolved proteins were transferred to a nitrocellulose membrane for $2 \mathrm{~h}$ at $40 \mathrm{~V}$. Each membrane was incubated separately with the primary antibody: anti-MMP-1 (SC-30069; Santa Cruz Biotechnology, Inc., CA, USA), anti-MMP-9 (SC-10737; Santa Cruz Biotechnology, Inc.), anti-collagen-1 (ab292; Abcam, Cambridge, UK) and anti-actin (A5316; Sigma-Aldrich, Saint Louis, MO, USA) overnight at $4^{\circ} \mathrm{C}$. The membranes were washed with a washing buffer (137 mM NaCl, $2.7 \mathrm{mM} \mathrm{KCl,} 10 \mathrm{mM} \mathrm{Na} \mathrm{HPO}_{4}, 2 \mathrm{mM}$ $\mathrm{KH}_{2} \mathrm{PO}_{4}$, and $0.05 \%$ Tween-20) and incubated with horse- 
Table I. Sources of sea buckthorn fruit blends.

Source

Vendor

Contents (\%)

Sea buckthorn fruit extract
Blueberry extract
Collagen
Pure natural honey
Hyaluronic acid
Total

Vitaworld Co., Korea

31.1

ESFood Co., Korea

25.4

Amorepacific Co., Korea

8.3

Shennong Honey Bio-Tech Co., Ltd., China

5.1

Bioland, Korea

0.1

70

radish peroxidase-conjugated goat anti-rabbit IgG (Zymed Laboratories, San Francisco, CA, USA) at a 1:1,000 dilution at room temperature for $2 \mathrm{~h}$. The membrane blots were developed using a Chemiluminescence Reagent Plus kit (Pfizer, New York, NY, USA).

Histological analysis and observation by optical microscopy. The skin tissues collected from the hairless mice were fixed with $10 \%$ formalin for $12 \mathrm{~h}$, embedded in paraffin wax, and sectioned into $4-\mu \mathrm{m}$ slices. The skin sections were then stained with hematoxylin and eosin (H\&E) (Sigma-Aldrich), and observed by optical microscopy. The thickness of the epidermis and dermis were measured using a Leica Application Suite (Leica Microsystems, Switzerland).

Activity analysis of superoxide dismutase (SOD). The SOD activity in the skin tissue was detected using a calorimetric assay and the reagents in the SOD Assay kit (Dojindo Molecular Technologies, Inc., Japan). First, the skin tissue $(100 \mathrm{mg})$ was homogenized in $600 \mu 1$ of sucrose buffer ( $0.25 \mathrm{~mol} / 1$ sucrose, $10 \mathrm{mmol} / 1 \mathrm{HEPES}, 1 \mathrm{mmol} / \mathrm{l}$ EDTA, $\mathrm{pH}$ 7.4) using a glass homogenizer. The lysate was harvested from the mixture by centrifugation at $10,000 \mathrm{x}$ g for $60 \mathrm{~min}$ and stored at $-70^{\circ} \mathrm{C}$ until needed for the enzyme activity assay. To measure the SOD activity, the sample lysate was diluted with the dilution buffer or saline as follows: $1,1 / 5,1 / 5^{2}, 1 / 5^{3}$, $1 / 5^{4}, 1 / 5^{5}, 1 / 5^{6}$. Sample solution $(25 \mu \mathrm{l})$ was aliquoted into a well of 96-well plate for each blank or sample, and $200 \mu 1$ of the WST working solution was added. In addition, an enzyme working solution $(20 \mu \mathrm{l})$ was added to each sample per well and mixed thoroughly. The enzyme reaction was induced by incubating the mixture plate at $37^{\circ} \mathrm{C}$ for $20 \mathrm{~min}$, and the absorbance was measured using a spectrophotometer at $450 \mathrm{~nm}$. The SOD activity was calculated directly using the following equation: SOD activity (inhibition rate $\%)=\left[\mathrm{A}_{\text {blank } 1}-\mathrm{A}_{\text {blank } 3}\right)$ - $\left.\left(\mathrm{A}_{\text {sample }}-\mathrm{A}_{\text {blank } 2}\right)\right] /\left(\mathrm{A}_{\text {blank 1 }}-\mathrm{A}_{\text {blank } 3}\right) \times 100\left(\mathrm{~A}_{\text {blank } 1}\right.$, absorbance of blank 1; $A_{\text {blank } 2}$, absorbance of blank 2; blank 3, absorbance of blank $3 ; \mathrm{A}_{\text {sample }}$, absorbance of sample).

Serum biochemical analysis. After the final day of drinking the vehicle, Vit and SFB, all mice were fasted for $24 \mathrm{~h}$ and blood was collected from the abdominal vein. Serum was obtained by centrifuging the blood after incubation for $30 \mathrm{~min}$ at room temperature. Serum biochemical components were assayed using a model 747 automated serum analyzer (Hitachi, Tokyo, Japan). All assays were measured with fresh serum using standard enzymatic methods. The measurements were conducted in duplicate.

Statistical analysis. One-way ANOVA was used to determine the significant differences between the no-radiation group and the UV radiation groups (SPSS for Windows, Release 10.10, Standard Version, Chicago, IL, USA). In addition, differences in the responses of the vehicle-treated group and Vit or SFB-treated group in the UV radiation group were evaluated using a post-hoc test (SPSS for Windows, Release 10.10, Standard Version) of the variance and significance levels. All values are reported as the mean \pm SEM. A P-value of $<0.05$ was considered significant.

\section{Results}

Effects of SFB on body weight and serum biochemical indicators. The changes in the body weight and serum biochemical indicators in the hairless mice were detected to determine the animal toxicity of SFB. Although the body weights of all groups were slightly lower at 4 weeks, there were no significant differences in body weight between the treated groups at 6 weeks (Fig. 1A). The hepatotoxicity of SFB was determined by measuring the alkaline phosphatase (ALP), alanine transaminase (ALT) and aspartate transaminase (AST) concentrations. The ALP concentration was decreased only slightly in the vehicle and SFB50-treated groups compared to the no-irradiation group, even though the other groups had constant levels (Fig. 1B). In addition, a decreasing pattern for the ALT concentration was detected in all irradiation groups except for the Vit-treated group (Fig. 1C). In the case of AST, however, a significant increase was observed only in the Vit- and SFB50-treated groups (Fig. 1D). Furthermore, the concentration of BUN and creatine (CRE), which indicate kidney toxicity, showed a different pattern (Fig. 1E and F). The BUN concentration was lower in the UV radiation group than in the no radiation group, even though the concentration in the Vit-treated group was slightly higher than that in the vehicle-treated group. A significant increase in CRE concentration was detected only in the SFB30-treated group. These results support the suggestion that Vit and SFB50 have low toxicity to the liver, even though SFB30 was nontoxic to the liver of mice. On the other hand, SFB30 showed lower toxicity to the kidneys of hairless mice.

Inhibition of $U V$-induced wrinkle formation by $S F B$ on the dorsal skin of hairless mice. The depth and number of wrinkles 
A
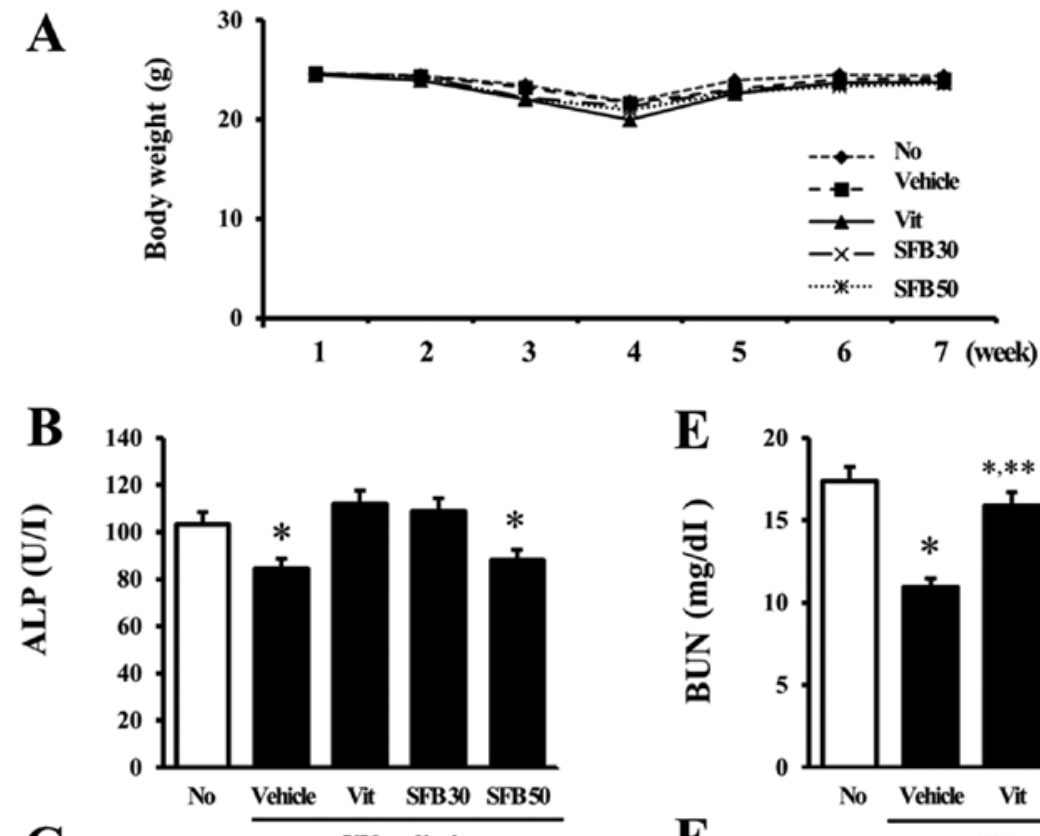

C UV radiation
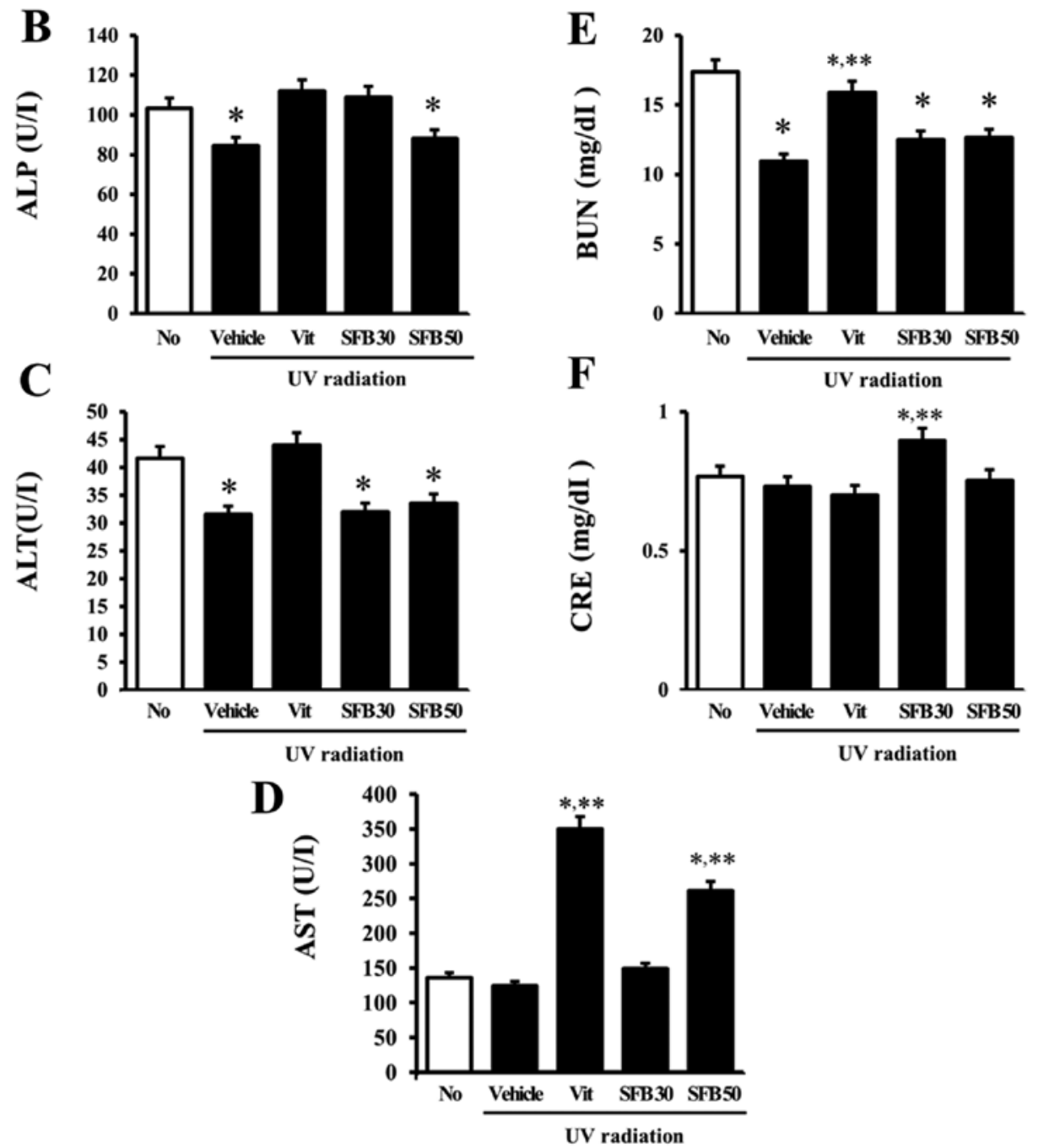

Figure 1. Effects of SFB on the body weight of ICR mice. (A) After administration of various solutions containing SFB to the hairless mice, the body weight was measured using an electronic balance every day. The concentrations of (B) ALP, (C) ALT, (D) AST, (E) BUN and (F) creatinine in the blood serum were analyzed in triplicate using a serum biochemical analyzer, as described in Materials and methods. The data are reported as the mean \pm SD from three replicates. ${ }^{*} \mathrm{P}<0.05$, significance level compared to the no-radiation group. ${ }^{* *} \mathrm{P}<0.05$, significance level compared to the vehicle-treated group.

were measured in the vehicle- or SFB-treated mice after 6 weeks of treatment to determine if SFB treatment can inhibit wrinkle formation induced by UV radiation. After UV radiation, the depth and number of wrinkles were significantly higher in the vehicle-treated group than in the no-irradiation group. On the other hand, there were significantly fewer wrinkles induced by UV radiation in the Vit-, SFB30- and SFB50-treated groups (Fig. 2A and B). In particular, the largest decrease was detected in the SFB50-treated group. Therefore, the consumption of Vit and SFB can effectively inhibit wrinkle formation on the dorsal skin of hairless mice.
Effects of SFB on the epidermis and dermis of hairless mice. UV radiation on dorsal skin of mice induced a significant change in skin histology (21). Therefore, the effects of the oral intake of SFB on the epidermis and dermis of mice skin were studied. After UV radiation, the epidermis and dermis were significantly thicker in the vehicle-treated group than the no-irradiation group. The number of adipocytes in the subcutaneous region was also significantly higher in the vehicle-treated group (Fig. 2C). On the other hand, these were slightly lower in the Vit- and SFB30-treated group than that in vehicle-treated group. The largest decrease was detected in the 
$\mathbf{A}$

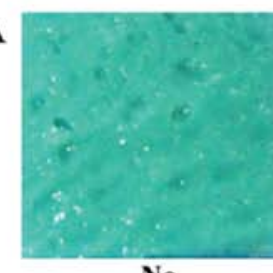

No

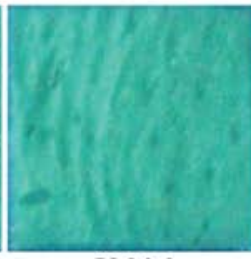

Vehicle

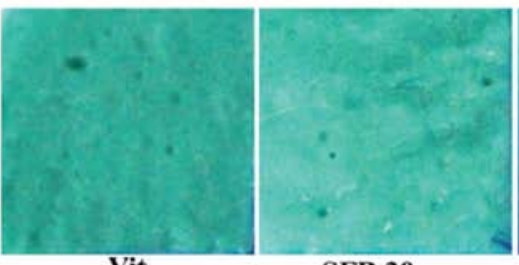

SFB 30

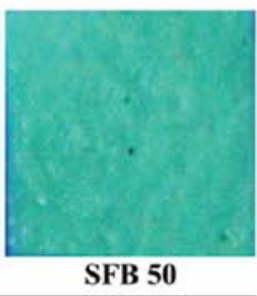

UV radiation
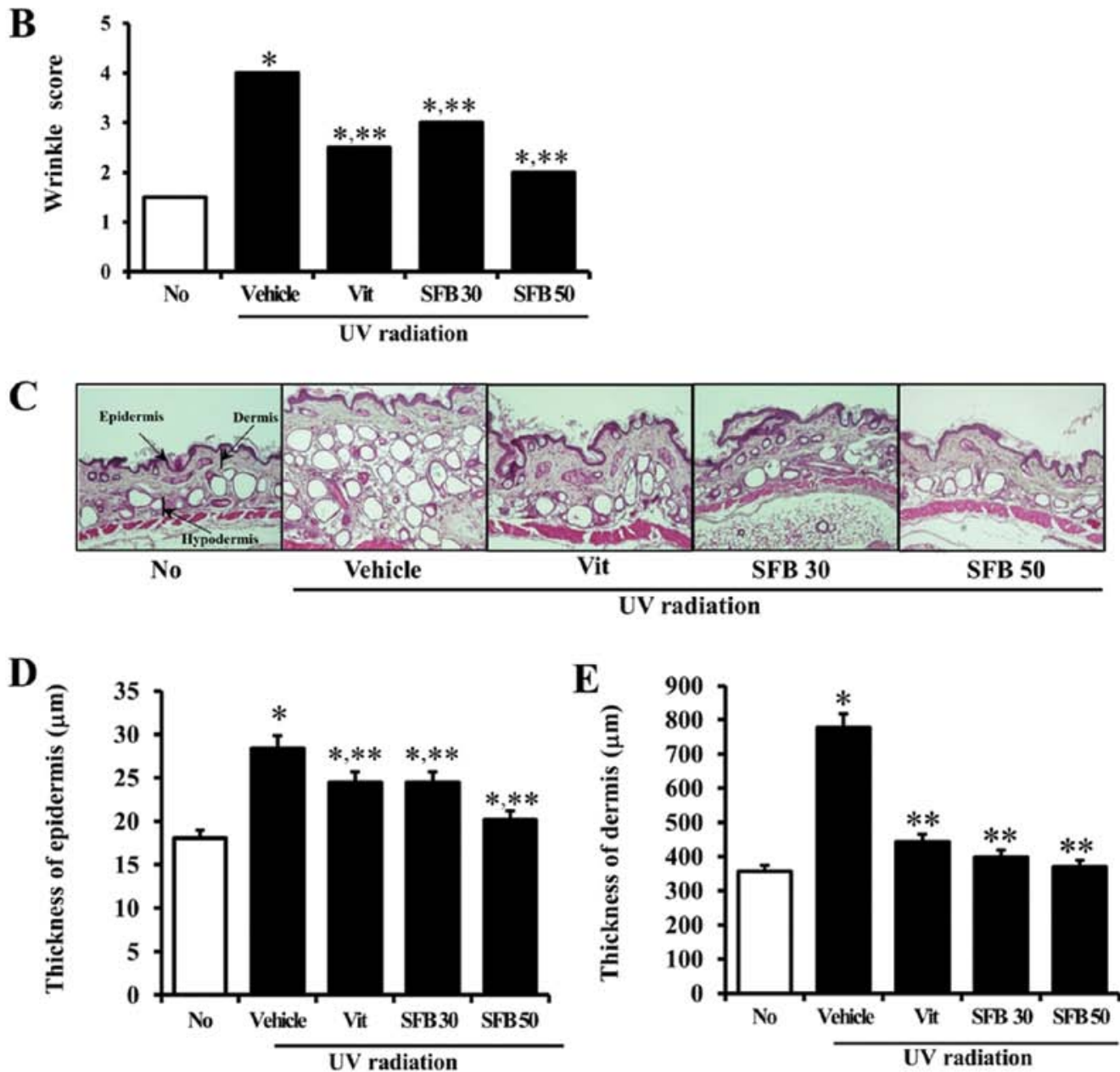

Figure 2. Inhibition of UV-induced wrinkle formation and histological alteration by SFB in the hairless mice. (A) Wrinkle formation was measured by replica grading at the final week. The level of wrinkles was assessed using the scoring system suggested by Bissette et al (18) (grade 0, no wrinkles; grade 1, a few shallow wrinkles; grade 2, some wrinkles; grade 3, several deep wrinkles). (B) The dorsal skin of hairless mice was prepared on a histological slide, and the skin thickness was measured from the epidermis to dermis. Cellular morphology was viewed at $x 20$ magnification. The data are presented as the mean \pm SD of three experiments. ${ }^{*} \mathrm{P}<0.05$ is the significance level compared to the no-radiation group. ${ }^{* *} \mathrm{P}<0.05$ is the significance level compared to the vehicle-treated group.

SFB50-treated group (Fig. 2D and E). Furthermore, all treated groups (Vit, SFB30 or SFB50) showed a similar number of adipocytes to the no-irradiation group (Fig. 2C). These results suggest that the oral intake of Vit, SFB30 or SFB50 for 6 weeks can induce a decrease in the thickness of epidermis and dermis, and the number of adipocytes.

Effects of SFB on the intact skin. To determine the effects of SFB oral intake on intact skin, the changes in transdermal water loss (TEWL), skin moisture content and erythema dose were measured after UV radiation for 6 weeks. The level of TEWL was significantly higher in the mice exposed to UV light when this level was compared with that of the no-radiation group. In the case of the Vit, SFB30 or SFB50 treatment, the level was lower than that of the vehicle-treated group. In particular, the level of TEWL in the SFB50-treated group was reduced to the level of the no-radiation group (Fig. 3A). In addition, the skin moisture content showed an opposite pattern to TEWL in the UV radiation group. The vehicle-treated group showed a lower skin moisture content than the no-radiation group. On the other hand, their level was increased significantly in the Vit, SFB30 and SFB50 group. The greatest increase was observed in the SFB50 group, followed by the SFB30 and Vit-treated groups (Fig. 3B). Nevertheless, there were no significant differences between the groups in terms of the erythema dose at 6 weeks (Fig. 3C). These results showed that SFB can help maintain the water content in the skin of hairless mice by preventing water loss and enhancing water retention. 
A

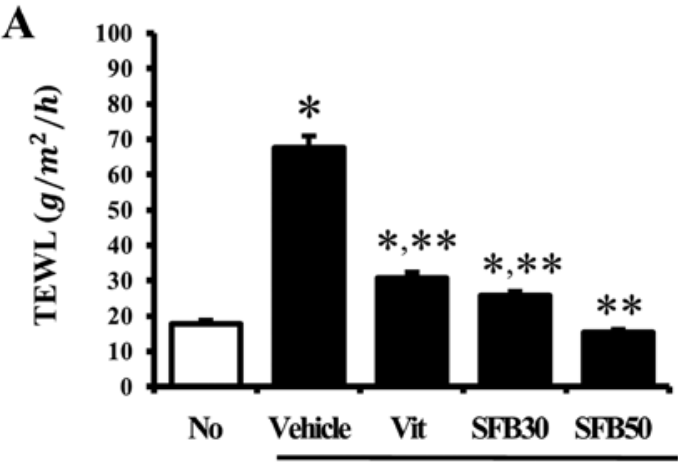

B

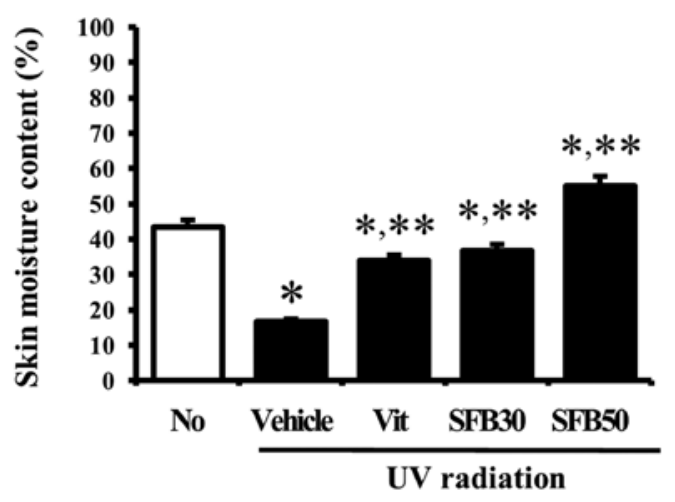

C

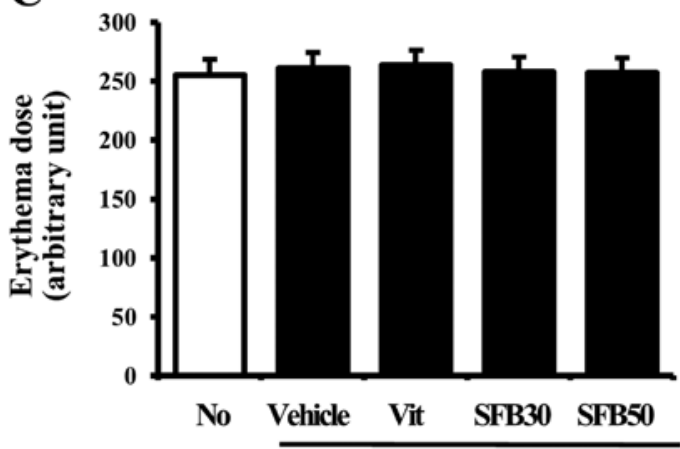

UV radiation

Figure 3. Effect of SFB oral intake on the intact skin. (A) The TEWL, (B) skin moisture content and (C) erythema dose in the dorsal skin of hairless mice were analyzed in triplicate using the appropriate probe, as described in Materials and methods. The data represents the mean \pm SD of three replicates. ${ }^{*} \mathrm{P}<0.05$ is the significance level compared to the no-radiation group. ${ }^{* *} \mathrm{P}<0.05$ is the significance level compared to the vehicle-treated group.

Effect of SFB oral intake on MMP expression. The level of MMP expression is increased by a range of factors including UV radiation, oxidative stress and cytokines (22). Accordingly, the level of MMP-1 and -9 proteins was examined by western blot analysis to determine if SFB oral intake can reduce MMPs expression. In the vehicle-treated group, the level of MMP-1 expression was significantly higher than that of the no-radiation group. MMP-1 expression, however, was decreased only in the SFB-treated group, whereas the level was maintained in the Vit-treated group (Fig. 4A). Furthermore, the expression pattern of MMP-9 was similar to MMP-1 except in the Vit-treated group. A high level of MMP-9 expression was detected in the vehicle-treated group and a significant decrease was observed
A
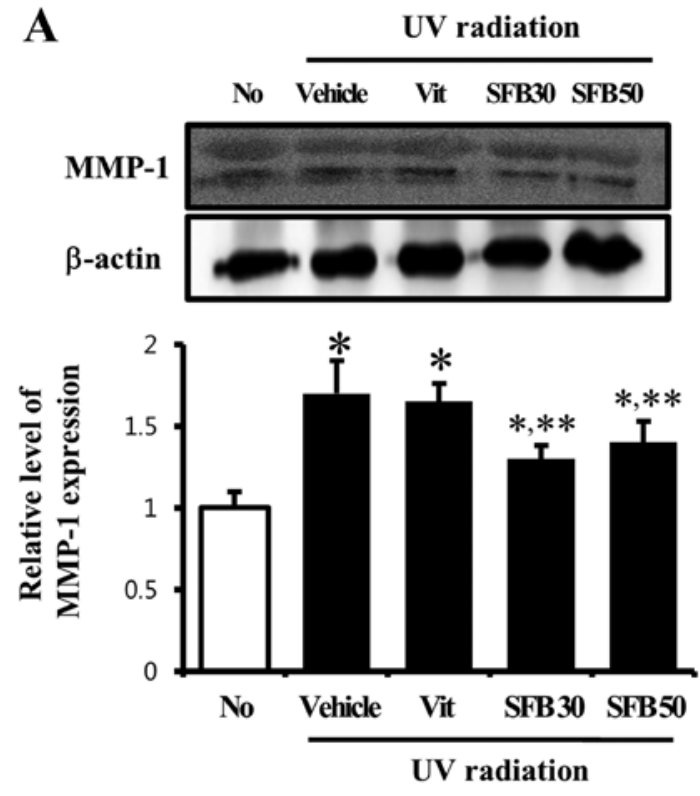

B
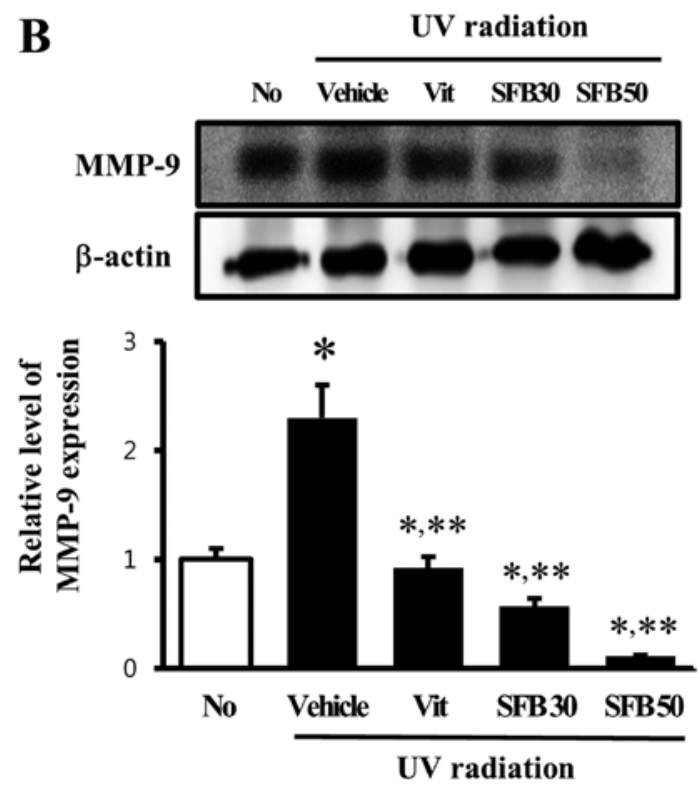

Figure 4. Effects of SFB oral intake on UV-induced (A) MMP-1 and (B) MMP-9 expression using western blotting. The total cell lysate were prepared from the skin tissues of the no-radiation, vehicle, Vit, SFB30 and SFB50 group, as described in Materials and methods. Fifty micrograms of protein per sample were immunoblotted with the antibodies for each protein. Three samples were assayed in triplicate using western blotting. The data is reported as the mean $\pm \mathrm{SD} .{ }^{*} \mathrm{P}<0.05$, significance level compared to the no-radiation group. ${ }^{* *} \mathrm{P}<0.05$, significance level compared to the vehicle-treated group.

in the SFB30 and SFB50-treated groups. In the case of the SFB50-treated group, the level of MMP-9 expression decreased by $70 \%$ (Fig. 4B). These results suggest that MMP expression induced by UV radiation can be reduced to normal by the oral intake of SFB.

Effect of SFB on collagen-1 content of skin tissue. MMPs are the only known mammalian enzymes capable of degrading a several types of collagen and gelatin (23). Therefore, this study examined whether the alteration of MMP-1 and -9 expression can be regulated the content of collagen-1 in skin tissue. Firstly, the collagen-1 level in the vehicle-treated group 

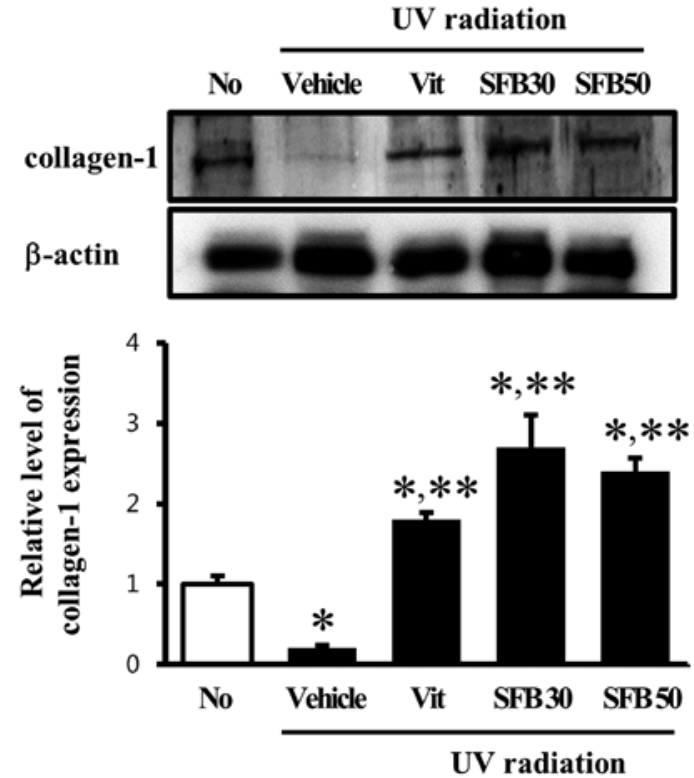

Figure 5. Effects of SFB oral intake on UV-induced collagen-1 expression by western blotting. The total cell lysate was prepared from the skin tissues of no-radiation, vehicle, Vit, SFB30 and SFB50 group, as described in Materials and methods. Fifty micrograms of protein per sample were immunoblotted with the antibodies for each protein. Three samples were assayed in triplicate using western blotting. The data is reported as the mean $\pm \mathrm{SD}$. ${ }^{*} \mathrm{P}<0.05$, significance level compared to the no-radiation group. ${ }^{* *} \mathrm{P}<0.05$, significance level compared to the vehicle-treated group.

was lower than in the no-radiation group. On the other hand, these levels in the Vit-, SFB30- and SFB50-treated groups were increased significantly ( 2 or 2.5 -fold) compared with the vehicle-treated group (Fig. 5). In the SFB30-treated group, the level of collagen-1 expression was similar to that detected in the SFB50-treated group. This suggests that the change in MMP-1 and -9 induced by UV radiation can induce a change in the collagen content in skin tissue.

Effects of SFB on the SOD activity of skin tissue. The effects of the oral intake of SFB on the antioxidant status of mice were examined by measuring the SOD activity in the skin tissue of the vehicle- and SFB-treated mice. A significant difference in SOD activity was not observed between the no-radiation group and vehicle-treated group. Furthermore, the SOD activity did not change in the Vit- and SFB30-treated groups. On the other hand, only the SFB50-treated group showed a high level of SOD activity compared to vehicle-treated group (Fig. 6). These results implicate SFB50 as a contributor to the increase in SOD activity in the skin of hairless mice after a 6 weeks treatment.

\section{Discussion}

Sea buckthorn is a wild shrub growing at high altitude in Asia and Europe and belong to the Elaeaganaceae family (24). All part of this plant are widely known as a rich source of biological active compounds such as flavonoids, carotenoids, steroids, vitamins, tannins and oleic acid $(25,26)$. The plant had been using to treat skin diseases, gastric ulcers, asthma and lung disorder for a long time (27). Especially, the leaf extracts, and fruit and seed oils of this plant have shown efficacy on

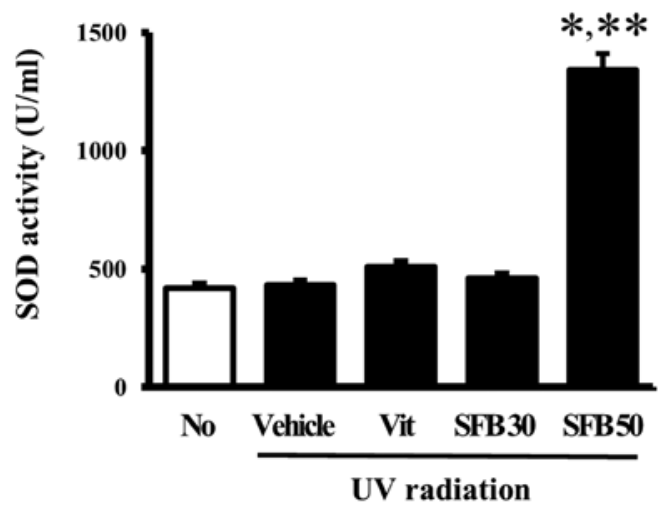

Figure 6. Effects of SFB oral intake on the SOD activity. The skin tissues were collected from the mice in the five groups treated with the vehicle, Vit or SFB solution. Five hairless mice per group were assayed in the SOD activity test. The data is reported as the mean $\pm \mathrm{SD}$ from three replicates. ${ }^{*} \mathrm{P}<0.05$, significance level compared to the no-radiation group. ${ }^{* *} \mathrm{P}<0.05$, significance level compared to the vehicle-treated group.

dermal wound healing and burns (28). However, there is no report on the effect of sea buckthorn fruit on photoaging. In this study, we investigated the function of sea buckthorn fruit on UV-induced skin aging. Our results showed that SFB had the ability to prevent skin aging through MMP-9 suppression and SOD activation.

The connective tissue in the skin is composed to various types of collagens, elastin, fibronectin, proteoglycan and other extracellular matrix (ECM) proteins. The normal structure of the connective tissue is maintained by a balance between the synthesis and degradation of the extracellular components. After UV radiation, the excessive deposition of the abnormal elastin complex and impairment of the collagen fibers were the most prominent features observed in the skin (16). Therefore, collagen is believed to be an important component to protect skin photodamage. As a part of an ongoing study of the collagen function, Zhuang et al (29) examined the effects of collagen (JC) and collagen hydrolysate (JCH) from Jellyfish on the mouse skin exposed to UV radiation. They focused on the abnormal changes in the antioxidative indicators, such as the SOD activity, glutathione peroxidase (GSH-Px) activity and catalase activity. The present study showed the effects of the SFB on of the change in skin morphology, water content, MMPs expression and collagen content. Our group previously reported that SFB should be considered as a potential therapeutic mixture for preventing and treating skin aging induced by UV radiation. Furthermore, these results provide evidence supporting the therapeutic potential of SFB as a novel therapeutic drug.

MMPs including MMP-1, -3 and -9 can degrade all types of ECM proteins, but can also process a number of bioactive molecules $(30,31)$. These enzymes participate in the degradation of the basement membrane and ECM $(23,32)$. In particular, MMP-1 plays important roles in the degradation of dermal collagens in the ECM, composed mainly of type-1 collagen during the aging of human skin (8). Therefore, an analysis of the MMP-1 expression level is a useful tool for screening anti-aging components $(33,34)$. In the present study, the level of MMP-1 expression was measured in the skin of mice in different groups. Only a significant change was detected in the SFB-treated group, but it was not changed in the Vit-treated 
group. This shows that SFB30 and SFB50 may work better on the anti-aging activity than a Vit solution. Therefore, SFB 30 and SFB 50 have similar effects to several compounds, such as tempol (35), compounds isolated from Fraxinus chinensis (14) and sulfur-containing cinnamate (15) reported in previous studies.

MMP-9 can degrade collagen type IV, which is an important component of the basement membrane during $\mathrm{UV}$ radiation in the skin (36). In addition, this protein has been associated with wound healing, tumor invasion and angiogenesis, and can induce the secretion of inflammatory cytokines (37,38). As shown Fig. 4, the level of MMP-9 expression in the vehicle-treated group was more than twice that observed in the no-radiation group. These results are similar to those of a previous study (5). Significant results were observed after the Vit, SFB30 and SFB50 treatments. In particular, SFB50 almost completely inhibited MMP-9 expression in the dorsal skin of hairless mice. Therefore, SFB is believed to be a strong candidate for inhibiting MMP-9 expression.

Collagen I and III are distributed abundantly in the dermis, and polymerized to produce extended mechanically stiff fibrils, which impart tensile strength to the tissue $(39,40)$. Therefore, wrinkle formation is correlated with the regulation of collagen synthesis and degradation (41). Most studies reported that the level of collagen in the skin is decreased significantly after UV radiation $(35,42)$. In this study, the collagen level in the radiation group was significantly lower than that in the no-radiation group. On the other hand, this level was recovered dramatically in the Vit-, SFB30- and SFB50-treated group. These changes were similar to those reported in previous studies examining the effects of tempol and retinoic acid on the collagen regulation of skin. The data first suggested that the expression of collagen might be affected more by the oral take of SFB30 and SFB50 than Vit.

Overall, these results suggest that SFB containing collagen, sea buckthorn fruit extract and blueberry extract can contribute to the protection and treatment of photodamaged skin caused by UV exposure.

\section{Acknowledgements}

This study was supported by a grant to PNU-Wellbeing Product Center from the Ministry of Knowledge Economy (B0011529).

\section{References}

1. Yaar M and Gilchrest BA: Photoageing: mechanism, prevention and therapy. Br J Dermatol 157: 874-887, 2007.

2. Langton AK, Sherratt MJ, Griffiths CEM and Watson REB: A new wrinkle on old skin: the role of elastic fibres in skin ageing. Int J Cosmet Sci 32: 330-339, 2010.

3. Warren R, Gartstein V, Kligman AM, Montagna W, Allendorf RA and Ridder GM: Age, sunlight, and facial skin: a histologic and quantitative study. J Am Acad Dermatol 25: 751-760, 1991.

4. Agache PG, Monneur C, Leveque JL and Derigal J: Mechanicalproperties and young's modulus of human-skin in vivo. Arch Dermatol Res 269: 221-232, 1980.

5. Choi HK, Kim DH, Kim JW, Ngadiran S, Sarmidi MR and Park CS: Labisia pumila extract protects skin cells from photoaging caused by UVB irradiation. J Biosci Bioeng 109: 291-296, 2010 .

6. Kim JH, Cho YH, Park SM, Lee KE, Lee JJ, Lee BC, Pyo HB, Song KS, Park HD and Yun YP: Antioxidants and inhibitor of matrix metalloproteinase-1 expression from leaves of Zostera marina L. Arch Pharm Res 27: 177-183, 2004.
7. Bergeron RJ, Huang G, Weimar WR, Smith RE, Wiegand J and McManis JS: Desferrithiocin analogue based hexacoordinate iron (III) chelators. J Med Chem 46: 16-24, 2003.

8. Brennan M, Bhatti H, Nerusu KC, Bhagavathula N, Kang S, Fisher GJ, Varani J and Voorhees JJ: Matrix metalloproteinase-1 is the major collagenolytic enzyme responsible for collagen damage in UV-irradiated human skin. Photochem Photobiol 78: 43-48, 2003.

9. Yasui $\mathrm{H}$ and Sakurai $\mathrm{H}$ : Chemiluminescent detection and imaging of reactive oxygen species in live mouse skin exposed to UVA. Biochem Biophys Res Commun 269: 131-136, 2000.

10. Hanson KM and Simon JD: Epidermal trans-urocanic acid and the UV-A-induced photoaging of the skin. Proc Natl Acad Sci 95: 10576-10578, 1998.

11. Ropke CD, da Silva VV, Kera CZ, Miranda DV, de Almeida RL, Sawada TC and Barros SB: In vitro and in vivo inhibition of skin matrix metalloproteinases by Pothomorphe umbellata root extract. Photochem Photobiol 82: 439-442, 2006.

12. Lee YS, Jin DQ, Beak SM, Lee ES and Kim JA: Inhibition of ultraviolet-A-modulated signaling pathways by asiatic acid and ursolic acid in HaCaT human keratinocytes. Eur J Pharmacol 476: 173-178, 2003.

13. Pallela R, Young NY and Kim SK: Anti-photoaging and photoprotective compounds derived from marine organisms. Mar Drugs 8: 1189-1202, 2010.

14. Lee BC, Lee SY, Lee HJ, Sim GS, Kim JH, Kim JH, Cho YH, Lee DH, Pyo HB, Choe TB, et al: Anti-oxidative and photoprotective effects of coumarins isolated from Fraxinus chinensis. Arch Pharm Res 30: 1293-1301, 2007.

15. Chiang CC, Chang TC, Tsai HJ and Hsu LY: Synthesis and biological evaluation of sulfur-containing cinnamate and salicylate derivatives. Chem Pharm Bull 56: 369-373, 2008.

16. Park CH, Lee MJ, Kim JP, Yoo ID and Chung JH: Prevention of UV radiation-induced premature skin aging in hairless mice by the novel compound Melanocin A. Photochem Photobiol 82: 574-578, 2006.

17. Nam SH, Jung SE, Lee YK, Kim JE, Lee EP, Choi HW, Kim HS, Lee JH, Jung YJ, Lee CY, et al: Topical application of selenium can significantly relieve UV-induced skin aging in hairless mice. Lab Anim Res 26: 37-45, 2010.

18. Bissett DL, Chatterjee R and Hannon DP: Photoprotective effect of topical anti-inflammatory agents against ultraviolet radiation-induced chronic skin damage in the hairless mouse. Photodermatol Photoimmunol Photomed 7: 153-158, 1990.

19. Tsukahara K, Moriwaki S, Fujimura T and Takema Y: Inhibitory effect of an extract of Sanguisorba officinalis L. on ultraviolet-Binduced photodamage of rat skin. Biol Pharm Bull 24: 998-1003, 2001.

20. Baba H, Masuyama A, Yoshimura C, Aoyama Y, Takano T and Ohki K: Oral intake of Lactobacillus helveticus-fermented milk whey decreased transepidermal water loss and prevented the onset of sodium dodecylsulfate-induced dermatitis in mice. Biosci Biotechnol Biochem 74: 18-23, 2010.

21. Koshiishi I, Horikoshi E, Mitani H and Imanari T: Quantitative alterations of hyaluronan and dermatan sulfate in the hairless mouse dorsal skin exposed to chronic UV irradiation. Biochim Biophys Acta 1428: 327-333, 1999.

22. Fisher GJ, Datta SC, Talwar HS, Wang ZQ, Varani J, Kang S and Voorhees JJ: Molecular basis of sun-induced premature skin ageing and retinoid antagonism. Nature 379: 335-339, 1996.

23. Lauer-Fields JL, Juska D and Fields GB: Matrix metalloproteinases and collagen catabolism. Biopolymers 66: 19-32, 2002.

24. Gupta A, Kumar R,Pal K, Singh V,Banerjee PK and Sawhney RC: Influence of sea buckthorn (Hippophae rhamnoides L.) flavone on dermal wound healing in rats. Mol Cell Biochem 290: 193-198, 2006.

25. Rousi A: The genus Hippophae L., a taxonomic study. Ann Bot Fenici 8: 177-227, 1971.

26. Beveridge T, Li TS, Oomah BD and Smith A: Sea buckthorn products: manufacture and composition. J Agric Food Chem 47: 3480-3488, 1999.

27. Suleyman H, Gumustekin K, Taysi S, Keles S, Oztasan N, Aktas O, Altinkaynak K, Timur H, Akcay F, Akar S, et al: Beneficial effects of Hippophae rhamnoides L. on nicotine induced oxidative stress in rat blood compared with vitamin E. Biol Pharm Bull 25: 1133$1136,2002$.

28. Gupta A, Kumar R, Pal K, Banerjee PK and Sawhney RC: A preclinical study of the effects of sea buckthorn (Hippophae rhamnoides L.) leaf extract on cutaneous wound healing in albino rats. Int J Low Extrem Wounds 4: 88-92, 2005. 
29. Zhuang Y,Hou H, Zhao X, Zhang Z and Li B: Effects of collagen and collagen hydrolysate from jellyfish (Rhopilema esculentum) on mice skin photoaging induced by UV irradiation. J Food Sci 74: 183-188, 2009.

30. Eisen AZ, Jeffrey JJ and Gross J: Human skin collagenase. Isolation and mechanism of attack on the collagen molecule. Biochim Biophys Acta 151: 637-645, 1968.

31. Lazarus GS, Daniels JR, Lian J and Burleigh MC: Role of granulocyte collagenase in collagen degradation. Am J Pathol 68: 565-578, 1972.

32. Visse R and Nagase H: Matrix metalloproteinases and tissue inhibitors of metalloproteinases: structure, function, and biochemistry. Circ Res 92: 827-839, 2003.

33. Pinnell SR: Cutaneous photodamage, oxidative stress, and topical antioxidant protection. J Am Acad Dermatol 48: 1-19, 2003.

34. Fisher GJ, Datta S, Wang Z, Li XY, Quan T, Chung JH, Kang S and Voorhees JJ: c-Jun-dependent inhibition of cutaneous procollagen transcription following ultraviolet irradiation is reversed by all-trans retinoic acid. J Clin Invest 106: 663-670, 2000.

35. Yan SX, Hong XY, Hu Y and Liao KH: Tempol, one of nitroxides, is a novel ultraviolet-A1 radiation protector for human dermal fibroblasts. J Dermatol Sci 37: 137-143, 2005.

36. Amano S, Ogura Y, Akutsu N, Matsunaga Y, Kadoya K, Adachi E and Nishiyama T: Protective effect of matrix metalloproteinase inhibitors against epidermal basement membrane damage: skin equivalents partially mimic photoageing process. Br J Dermatol 153: 37-46, 2005.
37. Kim S, Kim Y, Lee Y, Cho KH, Kim KH and Chung JH: Cholesterol inhibits MMP-9 expression in human epidermal keratinocytes and HaCaT cells. FEBS Lett 581: 3869-3874, 2007.

38. Onoue S, Kobayashi T, Takemoto Y, Sasaki I and Shinkai H: Induction of matrix metalloproteinase-9 secretion from human keratinocytes in culture by ultraviolet B irradiation. J Dermatol Sci 33: 105-111, 2003

39. Gosline J, Lillie M, Carrington E, Guerette P, Ortlepp C and Savage K: Elastic proteins: biological roles and mechanical properties. Philos Trans R Soc Lond B Biol Sci 357: 121-132, 2002.

40. Heim AJ, Matthews WG and Koob TJ: Determination of the elastic modulus of native collagen fibrils via radial indentation. Appl Phys Lett 89: 181902-181903, 2006.

41. Chen S, Kiss I and Tramposch KM: Effects of all-trans retinoic acid on UVB-irradiated and non-irradiated hairless mouse skin. J Invest Dermatol 98: 248-254, 1992.

41. Vicentini FT, Fonseca YM, Pitol DL, Iyomasa MM, Bentley MV and Fonseca MJ: Evaluation of protective effect of a water-in-oil microemulsion incorporating quercetin against UVB-induced damage in hairless mice skin. J Pharm Pharm Sci 13: 274-285, 2010 . 